\title{
Prevalence of Strongyle Infection and Associated Risk Factors in Horses and Donkeys in and around Mekelle City, Northern Part of Ethiopia
}

\author{
Wossene Negash $(\mathbb{D}$, Yosef Erdachew, and Teshager Dubie \\ College of Veterinary Medicine, Samara University, P.O. Box 132, Samara, Ethiopia \\ Correspondence should be addressed to Wossene Negash; tsgaw13@gmail.com
}

Received 21 December 2019; Revised 23 July 2020; Accepted 13 July 2021; Published 22 July 2021

Academic Editor: Nora Mestorino

Copyright (c) 2021 Wossene Negash et al. This is an open access article distributed under the Creative Commons Attribution License, which permits unrestricted use, distribution, and reproduction in any medium, provided the original work is properly cited.

\begin{abstract}
Background. In Ethiopia, equines serve in traction power, carting, recreation, festival packing, riding, transportation, and other activities since time immemorial. Strongyles are common equine health problems in Ethiopia though research based data on equine strongyles are limited particularly in the study areas, in and around Mekelle city. Therefore, the present study was intended to estimate the prevalence of common equine strongyles in and around Mekelle city from November 2018 to April 2019 and to assess risk factors associated with infection of strongyle parasites as well. Cross sectional design was used in this study, and the study population consisted of both donkeys and horses of all age and both sex groups. From randomly selected horses and donkeys, approximately 25 grams of faecal samples was drawn with gloved hands from rectum of study equines, labeled, and transported to laboratory for coprological examination. Flotation technique was employed to separate parasitic eggs from faeces, followed by microscopic examination for identification of strongyle eggs based on morphology. Pearson's chi-square $(\chi 2)$ was carried out to determine association between risk factors and parasitic infection. Moreover, both bivariate and multivariate logistic regression analyses were computed to assess the strength of association of those risk factors at $95 \% \mathrm{CI}$ and $P<0.05$. Result. Out of 384 samples collected, 204 were found to be positive for strongyles with an overall prevalence of 53.13\%. Prevalence of strongyle species in equines was also estimated to be $53 \%$ and $53.3 \%$ for donkeys and horses, respectively. Accordingly, of the six risk factors considered, only three factors (age, management type, and body condition scores) were found to influence the occurrence of strongyle infection and to be statistically significant as well. Conclusion. The higher prevalence of equine strongyles in the present study might be suggestive of urgent and coordinated actions to be in place.
\end{abstract}

\section{Introduction}

Ethiopia is endowed with immense natural resources occupying different agroecological zones and suitable environmental conditions making it a home for many livestock species and varying production systems. Recent estimated livestock population included 59.5 million cattle, 56.53 million heads of chicken, 30.70 million sheep, 30.20 million goats, 8.44 million donkeys, 2.16 million horses, 1.21 million camels, and 0.41 million mules [1]. Of the total population of equines, 364,940 are found in Tigray region [2]. Horses and donkeys are collectively named as equines. In Ethiopia, equines are used for packing, riding, carting, plowing/ traction power, and transportation [3]. Moreover, equines are also used for recreation and festivity specially horses. Equines play significant roles in agricultural systems of many developing countries holding magnificent share in food security and social equity in high food insecure nations, particularly in highly inaccessible areas $[4,5]$.

Though equines are known by their hardy characteristics, their prominent roles are impeded by health problems [6] of which the most common challenges lead to health problems, poor reproductivity and working performances, shorter lifespan, and finally death due to infectious diseases and parasitism [7]. Endoparasites are organisms that live within the body of the host [8]. Many internal parasites can 
infect equines including roundworms; mainly strongyles, flukes, tapeworms, protozoans, and fly larvae damage the intestine, respiratory system, and other internal organs of equines [9-11].

Strongyle nematodes are important equine gastrointestinal parasites belonging to the Superfamily Strongyloidea, family Strongylidae, genus Strongylus and comprising three species: Strongylus vulgaris, Strongylus edentatus, and Strongylus equinus. These live as adults in the large intestine of equids [12]. Equine strongyles are grouped into small and large strongyles. Large strongyles are more pathogenic relative to their smaller counterparts. Strongylosis is a devastating health problem particularly in young horses kept on permanent pastures although severe cases may occur in adults subjected to overcrowding and poor management [13, 14]. Strongylus vulgaris and Strongylus edentatus are the commonest equine health concerns in Ethiopia [15].

Being the most pathogenic species, Strongylus vulgaris is known as double tooth strongyle, whereas Strongylus edentatus is termed as toothless strongyle and Strongylus equinus known as triple toothed strongyle. Relatively, the first is smaller than the second [16]. Though strongyle infections are of very much concern and economically important equine health problem in Ethiopia, generally, research works or scientific data pertaining to equine strongyles are scarcely available to date particularly in and around Mekelle city. Therefore, the objectives of the current study were to estimate the prevalence of horses and donkeys strongyle infection in and around Mekelle city and to assess associated risk factors with the disease.

\section{Materials and Methods}

2.1. Description of the Study Areas. This cross sectional study was conducted in and around Mekelle city, from November 2018 to May 2019. Being the capital of Tigray region, Mekelle city is located $783 \mathrm{~km}$ from Addis Ababa in the northern eastern part of Ethiopia. Currently, the city has 7 subcities named as Hawelti, Hadnet, Ayder, Semen, Quiha, Kedamay Weyane, and Adi Haqi. Of the 7 subcities, Hadnet, Quiha, Adi Haqi, Kedamay Weyane, and Hawelti were chosen for the current study based on convenience. Mekelle city is located at a latitude of $13^{\circ} 20^{\prime} 50^{\prime \prime} \mathrm{N}$ and longitude of $39^{\circ} 32^{\prime}$ $38^{\prime \prime} \mathrm{E}$ with an altitude range of $2150-2270$ meter above sea level with arid to semiarid weather condition. The mean annual rainfall and temperature of the city were estimated at $200 \mathrm{~mm}$ to $11200 \mathrm{~mm}$ and $12.5^{\circ} \mathrm{C}$ to $27.5^{\circ} \mathrm{C}$, respectively [17]. Tigray region covers an area of $54,548.32 \mathrm{~km}^{2}$ and has an estimated livestock population of 3,596,649 cattle, 1,646,752 goats, 1,064,501 sheep, 364,940 equines, 13,661 camels, and $2,570,833$ poultry birds $[1,2]$.

2.2. Study Population. The study was conducted on donkeys and horses admitted to veterinary hospitals and city clinics in and around Mekelle city. The study included donkeys and horses of all age, clinically suspected, and apparently healthy groups as well as both sex groups.
2.3. Sampling Method and Sample Size Determination. Both the surrounding rural kebeles and Mekelle subcities were selected purposively. However, individual animal owners of study units (animals) were chosen using simple random sampling technique. To estimate the total number of animals to be included in the study, Thrusfield [18] formula was employed:

$$
N=\frac{(1.96) \cdot P \exp (1-P \exp )}{d^{2}},
$$

where $N$ is the sample size, 1.96 is the the value of " $Z$ " at $95 \%$ confidence interval, $P_{\exp }$ is the expected prevalence, and $d$ is the desired absolute precision.

By taking 95\% confidence interval, 5\% level of precision, and $50 \%$ expected prevalence into consideration in the above formula, the total sample size needed for the study was calculated to be 384 (200 donkeys and 184 horses). Study animals were categorized into three groups based on Loch and Bradley (1998). Accordingly, equines aged less than three years were considered as young, those aged between 3 and 10 ears as adult, and lastly equines aged more than 10 years as old.

\subsection{Sample Collection and Laboratory Analysis Method.} With gloved hands, approximately 25 grams of faecal samples was withdrawn from the rectum of restrained animals into screw capped universal sample bottle at the study sites. Following collection, each sample was uniquely labeled using each animal's identification parameters (species, age, sex, origin/site, collector's name, body condition score, and date of collection). Collected samples were packed and transported to Mekelle University Veterinary Parasitology Laboratory for immediate identification of strongyle parasites based on egg morphology.

2.5. Parasitological Techniques. For separation of parasite eggs from the faecal debris, flotation technique was applied. Technically, approximately $3 \mathrm{gm}$ of faeces was measure and placed into glass beaker followed by addition of $40 \mathrm{ml}$ flotation fluid, specifically magnesium sulfate solution. The mixture was continuously stirred using a glass rod. The dissolved suspension was then strained using tea strainer into another beaker. Then, the suspension was transferred to another test tube to an extent of meniscus formation at the top of the tube, and cover slip was gently placed over the meniscus and allowed to stand for 20 min. Finally, the cover slip to which the supernatant fluids were adhered was carefully lifted off the test tube, placed on microscopic slide, and examined under low power microscopic magnification (10x) (William, 2001). Strongyle species were identified based on oval morphology under the microscope.

In principle, flotation fluid was used to concentrate and separate eggs from most faecal debris. Since strongyle eggs have less specific gravity compared to flotation fluids (magnesium sulfate, sugar, sodium nitrate, and zinc sulfate), eggs floated and concentrated at the upper portion of the test tube containing sample mixture. On the other hand, faecal 
debris with higher gravity compared to the eggs concentrated at the lower portion of the test tube. Therefore, examination of the solution in the topmost layer clearly indicated the presence of strongyle parasite eggs. Hence, flotation method relies on the differences in the specific gravity of the eggs, faecal debris, and flotation solution. The flotation method is technically easy to process and faster as well. The disadvantage of most flotation techniques is that the walls of eggs and cysts can often collapse, thus hindering identification [19].

2.6. Data Management and Analysis. All relevant data were collected, coded, and entered precisely into Microsoft Excel application. Data were analyzed using STATA 14.0. Descriptive statistics was used to calculate prevalence and percentages whereas Pearson chi-square test $(\chi 2)$ was employed to assess association between parasite infection and risk factors. Both bivariate and multivariate logistic regression analyses were computed to measure the strength of association between strongyle infection and risk factors with $95 \% \mathrm{CI}$ and $P$ value less than 0.05 .

\section{Results}

Of 384 animals examined, 204 were found to be positive for gastrointestinal equine strongyles in both species with an overall prevalence of $53.13 \%$ (Table 1). Moreover, descriptive statistics has indicated the proportion of positive animals for species of equines. Accordingly, of the 204 animals positive for the parasites under study, 106 (53\%) were donkeys and 98 (53.3\%) were horses. The magnitude of parasitism was found to be approximately equivalent in both species. The study has also indicated that parasitic burdens were more in male as compared to female animals. Similarly, more parasitic intensity has been found in extensive type of management compared to semi-intensive management type. Both descriptive statistics and association analyses results are summarized in Table 1.

By applying 95\% CI and $P<0.05$, chi-square $(\chi 2)$ analysis was computed and indicated that only three (3) factors, namely, age, body condition, and management type, were significantly associated with strongyle infection $(P<0.05)$ (Table 1). However, sex, equine species, and origin of animals had no significant association with strongyle infection $(P>0.05)$ (Table 1).

To assess the degree of influence each factor could have, keeping other factors constant, on the infection of strongyles, bivariate logistic regression analysis was computed. Accordingly, age, body condition, and management type were found to have statistically significant association with strongyle parasite infection using 95\% CI and $P$ value 5\% (Table 2). Similarly, to assess the combined/simultaneous influence of all factors on strongyle infection, occurrence was computed employing multivariate logistic regression analysis with 95\% CI and $P<0.05$ (Table 2). In multivariate logistic regression analysis, adjusted odds ratio (AOR) was used. In age analysis, only adult equines had shown significant contribution $(P<0.000)$ to the overall significance of age impact. Adult equines were $63 \%$ less likely at risk as compared to old ones. In body condition analysis, animals with poor and medium body conditions were 6.99 times and 6.13 times more likely at risk as compared to good body conditioned animals, respectively. Similarly, management type was analyzed, and semi-intensively managed equines were 2.55 times more likely at risk as compared to extensively managed ones.

\section{Discussion}

Gastrointestinal (GI) parasite infection has a direct effect on the health and production potential of working equines, which contributes to the reduction in their work output and, ultimately, in the income of the owner and the community [20]. In this study, the overall estimated prevalence of strongyles in horses and donkeys was 53.13\% $(n=204 / 384)$ in and around Mekelle city on the basis of coprological microscopic examination results. The current study finding closely agreed with previous reports of Ashenafi et al. [21], who reported $47.4 \%$ in horse and donkeys in and around Kombolcha town, Ethiopia, and similarly Pandey and Eysker [22], who reported a prevalence of $48 \%$ in donkeys in Zimbabwe. On the other hand, the current finding was found to be lower as compared to the reports of Molla et al. $[23,24]$, who reported a prevalence of $64.61 \%, 100 \%, 99 \%$, and $100 \%$ for Menz Keya Gerbil District, East Shewa, and Adaa and Akaki of East Shewa, respectively. The nutritional status can affect the host immunity level, which means if the study animals access good nutrition, they would have enhanced immunity that can protect the host from parasite infection. On the other hand, if the study animals are malnutrioned, their immunity status would be decreased so that the study animals can be easily affected [25].

Moreover, the present finding was observed to be higher than the findings of Singh et al. [26], who reported a prevalence of $27.33 \%$ in Punjab and India, and Disassa et al. [25], who reported $5.37 \%$ in and around Dangila town, Ethiopia. These observed variations could be attributed to a difference in parasite epidemiology, managemental difference, lack of donkey sanctuary, absence of regular deworming service, and restriction on pasture grazing. In the current study, the prevalence of strongyles in horses and donkeys was estimated to be $53.3 \%$ and $53 \%$, respectively, and the degree of strongyle infection seems to be slightly higher in horses compared to donkeys with no statistical difference $(P>0.05)$.

The current finding closely agreed with the reports of $\mathrm{Ma}$ and wubit [12], who reported the prevalence of strongyle infections to be $44.55 \%$ in donkeys and $48.2 \%$ in horses. However, the current study revealed a higher prevalence at species level than Disassa et al. [25], who reported 5.82\% in donkeys and $4.92 \%$ in horses in and around Dangila town. On the other hand, the current study result was lower than the reports of Getachew et al. [24] from East Shewa-Adaa that revealed $100 \%$ and $99 \%$ in donkeys and in horses and Hassan et al. [27], who reported a higher prevalence of 99.15\% in Sudan. Moreover, the current result was lower than that reported by Feseha et al. [20] (100\% in donkeys) 
TABLE 1: Prevalence and risk factor analysis results.

\begin{tabular}{|c|c|c|c|c|c|}
\hline Risk factor & Number of examined & Number of positive & Prevalence & COR (95\% CI lower-upper) & $P$ value \\
\hline \multicolumn{6}{|l|}{ Species } \\
\hline Donkeys & 200 & 106 & 53 & & \multirow{2}{*}{0.959} \\
\hline Horses & 184 & 98 & 53.3 & $0.990(0.663-1.478)$ & \\
\hline \multicolumn{6}{|l|}{ Sex } \\
\hline Male & 351 & 189 & 53.85 & & \multirow{2}{*}{0.357} \\
\hline Female & 33 & 15 & 45.5 & $1.4(0.684-2.867)$ & \\
\hline \multicolumn{6}{|l|}{ Age } \\
\hline Young & 48 & 20 & 41.7 & & \multirow{3}{*}{0.003} \\
\hline Adult & 206 & 126 & 61.2 & $0.454(0.240-0.859)$ & \\
\hline Old & 130 & 58 & 44.62 & $0.88(0.45-1.73)$ & \\
\hline \multicolumn{6}{|l|}{ Body condition } \\
\hline Good & 130 & 57 & 43.85 & & \multirow{3}{*}{$P \leq 0.001$} \\
\hline Medium & 166 & 75 & 45.18 & $0.947(0.597-1.504)$ & \\
\hline Poor & 88 & 72 & 81.82 & $0.174(0.091-0.330)$ & \\
\hline \multicolumn{6}{|l|}{ Management } \\
\hline Extensive & 265 & 158 & 59.62 & & \multirow{2}{*}{$P \leq 0.001$} \\
\hline Semi-intensive & 119 & 46 & 38.66 & $2.343(1.50-3.65)$ & \\
\hline \multicolumn{6}{|l|}{ Areas (sites) } \\
\hline Hadnet & 103 & 52 & 50.49 & & \multirow{5}{*}{0.291} \\
\hline Quiha & 89 & 42 & 47.19 & $1.141(0.647-2.013)$ & \\
\hline Adi Haqi & 62 & 37 & 59.68 & $0.68(0.36-1.30)$ & \\
\hline Kedamay Weyane & 59 & 37 & 62.71 & $0.606(0.31-1.16)$ & \\
\hline Hawelti & 71 & 36 & 50.70 & $0.991(0.541-1.815)$ & \\
\hline Total & 384 & 204 & 53.13 & & \\
\hline
\end{tabular}

TABLe 2: Portrayal of logistic regression analysis results.

\begin{tabular}{|c|c|c|c|c|c|c|}
\hline \multirow{2}{*}{ Variable } & \multicolumn{2}{|c|}{ Test result } & \multicolumn{2}{|c|}{ Bivariate logistic regression } & \multicolumn{2}{|c|}{ Multivariate logistic regression } \\
\hline & Positive & Negative & COR $(95 \% \mathrm{CI})$ & $P$ value & AOR $(95 \% \mathrm{CI})$ & $P$ value \\
\hline \multicolumn{7}{|l|}{ Age } \\
\hline Young & 20 & 28 & 1 & & $1.063(0.510-2.213)$ & 0.871 \\
\hline Adult & 126 & 80 & $0.454(.240-0.859)$ & 0.015 & $0.370(0.225-0.611)$ & $P \leq 0.001$ \\
\hline Old & 58 & 72 & $0.887(0.454-1.733)$ & 0.725 & 1 & \\
\hline \multicolumn{7}{|l|}{ Body condition } \\
\hline Poor & 57 & 73 & 1 & & $6.990(3.542-13.793)$ & $P \leq 0.001$ \\
\hline Medium & 75 & 91 & $0.947(0.597-1.504)$ & 0.819 & $6.131(3.191-11.781)$ & $P \leq 0.001$ \\
\hline Good & 72 & 16 & $0.174(0.091-0.330)$ & $P \leq 0.001$ & 1 & \\
\hline \multicolumn{7}{|l|}{ Managements } \\
\hline Extensive & 158 & 107 & 1 & & 1 & \\
\hline Semi-intensive & 46 & 73 & $2.343(1.505-3.65)$ & $P \leq 0.001$ & $2.552(1.571-4.147)$ & $P \leq 0.001$ \\
\hline
\end{tabular}

and those reported by Tolla et al. [28] in and around Gondar, Ethiopia, Tesfu et al. [29] in and around Hawassa town, and $\mathrm{Ra}$ and Etaferahu [11] in South Wollo zone $(87.81 \%, 76 \%$, $70.8 \%$ in donkeys and $66.67 \%, 64.9 \%, 58.5 \%$ in horses, respectively). In addition, the current study finding was lower as compared to previous reports by Mezgebu et al. [30], who reported $66.7 \%$ prevalence in Gondar Town, and Uslu and Guçlu [31], who reported $100 \%$ in Konya, Turkey. The observed variations could be attributable to agroecological difference, parasite load in the study areas, and equine population in the study sites. Furthermore, differences could be associated with hygienic conditions, regular deworming services, and reduced parasitism [26].

Statistically, the age of study animals and strongyle infection were significantly associated $(P<0.05)$. Similar outcome was observed by Dryden et al. [32] and Ibrahim et al. [33], who reported statistically significant difference between age groups with higher prevalence in adult and lower prevalence in young animals. The agreement of the findings could be associated with study design, technique applied, sample size, geographic conditions, work load, and management difference or husbandry practices.

The present study also estimated prevalence of equine strongylosis to be $45 \%$ in females and $54 \%$ in males with no significant difference $(P>0.05)$. Similar phenomenon was reported by Fikru et al. $[34,35]$. The absence of significant association between the parasite infection and sex might be due to communal grazing system, biological difference, anthelmintic treatment services, and husbandry practices. However, in previous study findings, Bereket et al. and 
Basaznew et al. [36, 37] have reported significant association between parasitic infection and sex. The significant association observed in these studies might be due to the fact that females are managed for breeding purpose and kept in home whereas male equines are used for packing purposes and carry wood a long distance to market places usually crossing different agroecological areas which might increase the probable exposure of males to strongyle infection as compared to their female counterparts.

The current study has also indicated a statistically significant difference $(P<0.05)$ between body condition scores and strongyle infections with infection rates of $81.82 \%$, $45.18 \%$, and $43.83 \%$ for good, medium, and poor body condition scores, respectively. The current study has reported a slightly higher prevalence related to body condition score compared to Mangassa and Tafese [38], who reported a significant association between body condition and parasite infection in and around Batu town with prevalence of $59.26 \%, 43.17 \%$, and $30.6 \%$ in poor, medium, and good body condition scores, respectively. However, the current study finding was lower as compared to the findings of Tesfu et al. [29], who reported $72.5 \%, 71.6 \%$, and $70.7 \%$ in poor, medium, and good body condition scores, respectively. Similarly, significant difference was reported by FAO and Francisco et al. $[39,40]$. The present study has also reported prevalence of equine strongyle infection in study sites of $50 \%, 47 \%, 60 \%, 63 \%$, and 51\% for Hadnet, Quiha, Adi Haqi, Kedamay Weyane, and Hawelti, respectively, with no statistically significant difference $(P>0.05)$ between the study sites and parasite occurrence.

Under the current study, management type was found to have a statistically significant difference $(P<0.05)$ with strongyle infection in horses and donkeys revealing infection rates of $60 \%$ and $39 \%$ for extensive and semi-intensive management systems, respectively. However, the current study has reported a lower infection rate than that reported by Mezgebu et al. [27], $83.33 \%$ and $80.43 \%$ in grazing and mixed feeding, respectively, in and around Gondar town, which could indicate that the prevalence of extensive management system is higher than semi-intensive management system, which could be related to husbandry practices, agroecology, hygienic condition, deworming services, study design, sample size, and analysis approach.

\section{Conclusion and Recommendations}

Strongyle infections are important health problems of equines in the study areas with an overall prevalence of $53.13 \%$. Factors such as species, age, body condition, and management type were found to be contributing risk factors in the parasite infection rate, whereas sex, species, and origin of study animals were found to have nonsignificant difference with the parasite infection. In order to minimize losses attributed to the equine strongyle infection in the area, equine owners should be informed of the economic importance and methods of control and prevention of helminths of equines through management improvements. Furthermore, research should be encouraged on equine strongyle parasites and regular and strategic deworming programs, and efficacious anthelmintics should be administered regularly. Moreover, advanced epidemiological studies on equine strongyle need to be strengthened.

\section{Abbreviations \\ AOR: $\quad$ Adjusted odds ratio \\ CI: Confidence interval \\ COR: Crude odds ratio \\ CSA: Central statistic authority \\ TBOANR: Tigray Bureau of Agriculture and Natural Resources \\ GI: $\quad$ Gastrointestinal.}

\section{Data Availability}

The analysed data can be released if it is necessary and requested by the concerned body. For time being, for data security/copyright/safety purpose, the data are unavailable. So, the data used to support this study are available from the corresponding author upon request.

\section{Ethical Approval}

Ethical approval for this research study was obtained from Samara University, College of Veterinary Medicine of Animal Research Ethics and Review Committee. All efforts were made to minimize animal suffering during sample collection.

\section{Consent}

Before sample collection, equine owners were made aware of and informed about the study objectives and the type of sample to be collected, and they made verbal consent with the required knowledge and awareness. Thus, oral consent was obtained from the equine owners to take samples from their equine population for research purpose.

\section{Conflicts of Interest}

The authors declare that they have no conflicts of interest.

\section{Authors' Contributions}

Teshager Dubie and Wossene Negash have contributed to title approval, proposal writing guidance, material support, data analysis, paper writing, and manuscript preparation. Yosef Erdachew has contributed to proposal preparation and sample/data/laboratory analysis.

\section{Acknowledgments}

The authors thank Samara University, Mekelle University, and Mekelle city administration for strong and valuable material, laboratory facility aid, transportation, and guidance. 


\section{References}

[1] Central Statistical Agency of Federal Democratic Republic of Ethiopia, "Central statistical agency: agricultural sample survey 2017, CSA, Addis Ababa, Ethiopia," 2017.

[2] Tigray BoFED, "Bureau of finance and economic development of Tigray regional state, Mekelle Ethiopia," Bulletin, vol. 1, no. 35-45, 2008.

[3] M. Getachew, G. Feseha, A. Trawford, and S. W. J. Reid, "A survey of seasonal patterns in strongyle faecal worm egg counts of working equids of the central midlands and lowlands, Ethiopia," Tropical Animal Health and Production, vol. 40, no. 8, pp. 637-642, 2008.

[4] F. Yoseph, F. Mengistu, T. Teklu, Y. Firwe, and D. Betere, "Seasonal variation in the parasite burden and body condition of working donkeys in East Shewa and West Shewa Regions of Ethiopia," Tropical Animal Health and Production, vol. 39, pp. 34-43, 2008.

[5] M. H. Woodford, "Veterinary aspects of ecological monitoring: the natural history of emerging infectious diseases of humans, domestic animals and wildlife," Tropical Animal Health and Production, vol. 41, no. 7, pp. 1023-1033, 2009.

[6] W. Marquardt and R. Demaree, Parasitology and Vector Biology, Harcourt Academic Press, Cambridge, MA, USA, 2nd edition, 1999.

[7] E. Tamador, A. Ahmed, and M. Abdalla, "The role of donkeys in income generation and the impact of endo-parasites on their performance," Journal of Veterinary Medicine and Animal Production, vol. 2, no. 2, pp. 65-89, 2011.

[8] A. B. Lora, Veterinary Parasitology. The practical veterinarian, Vol. 53, Butterworth-Heinemann, Oxford, UK, 2001.

[9] J. Pereira and S. Vianna, "Gastrointestinal parasitic worms in equines in the paraíba valley, state of são paulo, Brazil," Veterinary Parasitology, vol. 140, no. 3-4, pp. 289-295, 2006.

[10] M. Taylor, R. Coop, and L. Waller, Veterinary Parasitology, Blackwell Science, Ltd., Oxford, England, 3rd edition, 2007.

[11] A. Ra and Y. Etaferahu, "Gastrointestinal parasites of equine in South Wollo zone, north eastern Ethiopia," Global Veterinaria, vol. 11, no. 6, pp. 824-830, 2013.

[12] B. Ma and T. Wubit, "Prevalence of strongyle infection and associated risk factors in horse and donkeys in and around Batu town, eastshoa, oromia regional state, Ethiopia," Food Science and Quality Management, vol. 54, pp. 2224-6088, 2016.

[13] T. Abayneh, B. Gebreab, Z. Ga, and A. Tadess, "The potential role of donkeys in land village in central Ethiopia," Bulletin of Animal's Health and Production in Africa, vol. 50, pp. 172$178,2002$.

[14] G. Admasu, A. Zelalem, and G. Ayele, "Prevalence of strongyle parasites in working horses in GobaWoreda, bale zone, Ethiopia," European Journal of Biological Sciences, vol. 6, no. 3, pp. 66-70, 2014.

[15] G. Urquhart, J. Arnovrm, J. Dincan, F. Jennings, and J. Duncan, Veterinary Parasitologypp. 42-47, Blackwell Science, London, UK, 2nd edition, 1996.

[16] S. Mandal, Veterinary Parasitology at a Glance, IBDC, Lucknow, India, 2 edition, 2012.

[17] TBONNR: Regional Animal Health Research Program Strategy Document, Tigray Bureau of Agriculture and Natural Resource (TBOANR), Tigray, Ethiopia, 1998.

[18] M. V. M. Thrusfield, Veterinary Epidemiology, Vol. 233, Blackwell Science, Singapore, 3rd edition, 2007.

[19] J. William, Veterinary Parasitology. A Reference Manual 5th edition, pp. 214-222, Blackwell, Singapore, 2001.
[20] G. Feseha, K. Alemu, I. Friew, Y. Abule, and A. Ketema, Donkey Utilisation And Management In Ethiopia.Acp-Eu Technical Centre For Agricultural And Rural Cooperation (CTA), Wageningen, Donkey, People and Development, Addis Ababa, Ethiopia, 1999.

[21] A. Ashenafi, E. Samuel, N. Ma, and B. Shiret, "Prevalence of gastrointestinal nematode parasitic infections of horses and donkeys in and around Kombolcha town," Journal of Scientific Research, vol. 10, pp. 228-234, 2015.

[22] V. Pandey and M. Eysker, "Internal parasites of donkeys from the high-veld of Zimbabwe," Veterinary Journal, vol. 21, pp. 27-32, 1990.

[23] B. Molla, Y. Worku, A. Shewaye, and A. Mamo, "Prevalence of strongyle infection and associated risk factors in equine in Menz Keya Gerbil District, North-Eastern Ethiopia," Journal of Veterinary Medicine and Animal Health, vol. 7, no. 4, pp. 117-121, 2015.

[24] M. Getachew, G. Trawford, G. Feseha, and J. Geid, "Gastrointestinal nematodes of donkeys (Equusasinus) in Khartoum state, Sudan," Journal of Animal Veterinary advances, vol. 3, pp. 736-739, 2010.

[25] D. Haimanot, A. Addise, Z. Tilahun, and K. Girma, "Prevalence of strongyle infection in horses and donkeys in and around Dangila town, northwest Ethiopia," Acta Parasitologica Globalis, vol. 6, no. 1, pp. 14-19, 2015.

[26] G. Singh, N. Singh, H. Singh, and S. Rath, "Assessment of risk factors associated with prevalence of strongyle infection in equines from central Plain Zone, Punjab," Journal of Parasitic, vol. 40, pp. 1-5, 2015.

[27] T. Hassan, M. Salih, and A. Abakar, "A surve y of gastrointestinal nematodes of donkeys," Journal of Animal and Veterinary Advances, vol. 3, pp. 736-739, 2004.

[28] M. Tolla, T. Ketema, and T. Firaol, "Prevalence of gastrointestinal parasites of horses and donkeys in and around gondar town," Open Journal of Veterinary Medicine, vol. 3, pp. 267-262, 2013.

[29] N. Tesfu, B. Asrade, R. Abebe, and S. Kasaye, "Prevalence and risk factors of gastrointestinal nematode parasites of horse and donkeys in Hawassa town, Ethiopia," Journal Veterinary Science Technology, vol. 5, pp. 156-166, 2014.

[30] T. Mezgebu, K. Tafess, and F. Tamiru, "Prevalence of gastrointestinal parasites of horses and donkeys in and around gondar town, Ethiopia," Open Journal of Veterinary Medicine, vol. 3 , no. 6 , p. $267,2013$.

[31] U. Uslu and F. Guclu, "Prevalence of endoparasites in horses and donkeys in Turkey," Bulletin-Veterinary Institute in Pulawy, vol. 51, p. 237, 2007.

[32] M. W. Dryden, P. A. Payne, R. Ridley, and V. Smith, "Comparison of common fecal flotation techniques for the recovery of parasite eggs and oocysts," Veterinary Therapeutics: Research in Applied Veterinary Medicine, vol. 6, pp. 15-28, 2005.

[33] N. brahim, T. Berhanu, B. Deressa, and T. Tolosa, "Survey of prevalence of helminth parasites of donkeys in and around Hawassa town, Southern Ethiopia," Global Veterinary, vol. 6, pp. 223-227, 2011.

[34] R. Fikru, S. Teshale, and M. Bizuneh, "Prevalence of equine gastrointestinal parasites in western highlands of oromia, Ethiopia," Bulletin of Animal Health and Production in Africa, vol. 53, pp. 161-166, 2005.

[35] S. Yoseph, D. Smith, A. Mengistu, F. Teklu, T. Firew, and Y. Befere, "Seasonal variation in the parasite burden and body condition of the working donkey in east shoa and west shoa 
zones of Ethiopia," Tropical Animal Health and Production, vol. 37, pp. 35-45, 1993.

[36] M. Bereket, W. Yalelet, and M. Alemgrzahu, "Prevalence of strongyle infections and associate $\mathrm{d}$ risk factors in equine in Menz keyagibril district, north eastern Ethiopia," Journal of Veterinary Medicine and Animal Health, vol. 7, no. 4, pp. 117-121, 2014.

[37] B. Basaznew, S. Zelalem, and C. Mersha, "Strongyle nematode infections of donkeys and mules in and around Bahirdar, Ethiopia," Global Veterinarian, vol. 9, no. 4, pp. 497-501, 2012.

[38] B. Mangassa and W. Tafese, "Prevalence of strongyle infection and associated risk factors in horse and donkeys in and around Batu town, eastshoa, oromia regional state, Ethiopia," Food Science and Quality Management, vol. 54, p. 69, 2016.

[39] FAO, Production Year Books, Vol. 158, FAO, Rome, Italy, 1996.

[40] I. Francisco, R. ArMorrondo, P. Sanchez-Andrade et al., "Intrinsic Factors influencing the infection by helminth parasites in horses under an oceanic climate Area (NW Spain)," JournalParasitol Research, vol. 2009, 2009. 